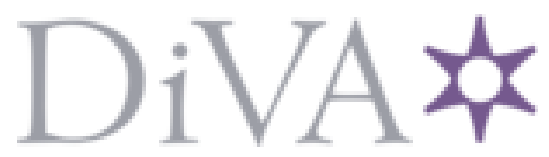

http://www.diva-portal.org

This is the published version of a paper published in Journal of Geophysical Research.

Citation for the original published paper (version of record):

Ronnmark, K., Hamrin, M. (2000)

Auroral Electron acceleration by Alfven waves and electrostatic fields.

Journal of Geophysical Research, 105(A 1 1): 25333-25344

http://dx.doi.org/10.1029/2000JA900103

Access to the published version may require subscription.

N.B. When citing this work, cite the original published paper.

Permanent link to this version:

http://urn.kb.se/resolve?urn=urn:nbn:se:umu:diva-85979 


\title{
Auroral Electron acceleration by Alfvén waves and electrostatic fields
}

\author{
Kjell Rönnmark and Maria Hamrin \\ Theoretical Space Physics, Umeå University, Umeå, Sweden
}

\begin{abstract}
We present a two-dimensional numerical model for the formation of discrete auroral arcs. This model describes the evolution of shear Alfvén waves generated by a growing force near the equatorial plane, and the transition to electrostatic fields when the force becomes stationary. The parallel electric fields on auroral field lines may be regarded as shear Alfvén waves driven by a magnetospheric generator at zero frequency. In our collisionless model, precipitating auroral electrons are accelerated to an energy of $350 \mathrm{eV}$ when the upward current is $3.1 \mu \mathrm{Am}^{-2}$. We also find that the electrostatic potential drop is proportional to the square of the current density.
\end{abstract}

\section{Introduction}

Discrete auroral arcs are perhaps the most spectacular phenomenon that regularly can be observed on the night sky. They have been studied intensely by space scientists for several decades, but as indicated in a review of 22 different theories for auroral arcs by Borovsky [1993], our understanding of the mechanisms behind the formation of discrete auroral arcs is still rather fragmented and uncertain.

Discrete arcs, in contrast to diffuse aurora, are characterized by the precipitation of field-aligned electrons with a rather well defined peak in their energy spectrum, and it is generally agreed that these electrons have been accelerated by a parallel electric field. Since the magnetospheric plasma is collisionless its parallel conductivity is expected to be very high, and any parallel electric fields should be short-circuited. Several theories have been proposed to explain how parallel potential drops reaching up to $10 \mathrm{kV}$ can exist along auroral field lines, in spite of the high conductivity. Anomalous resistivity caused by wave turbulence [e.g., Papadopolous, 1977] and electrostatic double layers [e.g., Block, 1972] have been suggested, but much of the discussion has recently revolved around two main groups of models for the parallel electric fields. One of these groups is purely electrostatic models, such as the kinetic models in which the magnetic mirror force prevents short-circuiting of the potential drop [Knight, 1973; Fridman and Lemaire, 1980; Janhunen and Olsson, 1998], and self-consistent fluid models [Mitchell et al., 1992; Ganguli et al., 1993; Rönnmark, 1999]. In the other group the parallel electric field associated with

Copyright 2000 by the American Geophysical Union.

Paper number 2000JA900103.

0148-0227/00/2000JA900103\$09.00 shear Alfvén waves is invoked to accelerate the auroral electrons [Goertz and Boswell, 1979; Haerendel, 1983; Lysak and Dum, 1983; Lysak, 1991; Kletzing, 1994; Streltsov and Lotko, 1995; 1999; Génot et al., 1999; Streltsov, 1999]. The model presented here combines features from these two groups into a common framework.

In this study we argue that the auroral display is powered by mechanical forces in the outer magnetosphere. These forces result from the braking of earthward plasma flows and the pressure gradients and anisotropies built up in this process. In a stationary state the forces drive an MHD generator that generates fieldaligned currents to and from the auroral ionosphere [e.g., Birn et al., 1999]. Information about changes in the driving force is transmitted by shear Alfvén waves that adjust the field-aligned currents. To carry the upgoing current from the ionosphere, downgoing electrons are accelerated to $\mathrm{keV}$ energies [Rönnmark, 1999], and the precipitation of these accelerated electrons causes the discrete aurora.

In our model a prescribed force is applied to the equatorial part of an auroral flux tube that initially is in static equilibrium. The equations defining the model are first derived, and the boundary conditions and parameter values used in this study are specified. In section 3 we follow the dynamic response of the flux tube in terms of electromagnetic fields, currents, and electron acceleration. Finally, we discuss some properties of the model and its implications for our understanding of auroral arc formation. Some numerical details are treated in Appendix A.

\section{The Auroral Circuit Model}

\subsection{Model Equations}

The geometry of the auroral current circuit and the generator region in the equatorial magnetosphere is 


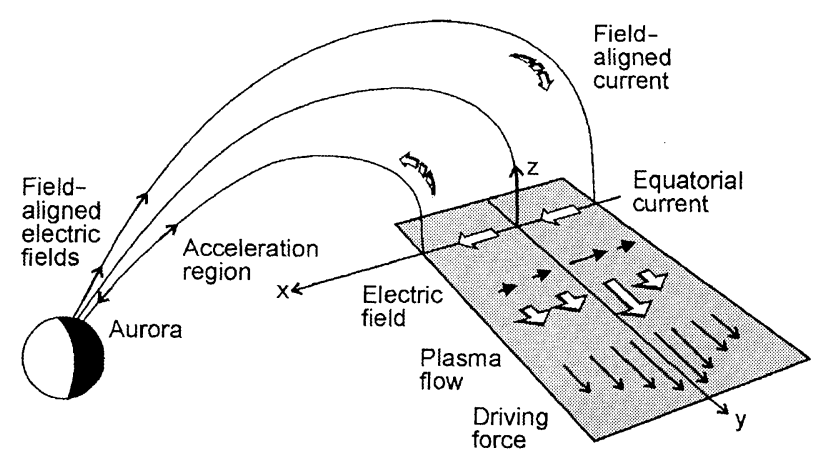

Figure 1. The geometry of the auroral current circuit and the generator region in the equatorial magnetosphere.

sketched in Figure 1. To derive our numerical model we introduce a coordinate system based on the flux tube geometry, with $z$ along the magnetic field lines, $x$ pointing to the earth and $y$ increasing to the west. The origin of these coordinates is at a point where an auroral field line crosses the equatorial plane. Since $x$ and $y$ are constant along field lines, a distance $d l$ is related to these coordinates by the metric

$$
d l^{2}=\left(d x^{2}+d y^{2}\right) \frac{B_{0}}{B_{z}}+d z^{2}
$$

where $B_{z}(z)$ is the local, time-independent, magnetic field and $B_{0}$ is the field strength at $z=0$ in the equatorial plane. We neglect the curvature of the field lines, and assume homogeneity in the $y$ direction. The current density $\mathbf{j}=j_{x} \hat{\mathbf{x}}+j_{z} \hat{\mathbf{z}}$ will then according to Maxwell's equations couple to the electric field components $E_{x}$, $E_{z}$, and the magnetic field $B_{y}$. Ampere's and Faraday's laws for these fields are in our coordinate system expressed as

$$
\begin{aligned}
\partial_{t} E_{x} & =-c^{2}\left(\partial_{z} B_{y}-B_{y} \frac{\partial_{z} B_{z}}{2 B_{z}}+\mu_{0} j_{x}\right) \\
\partial_{t} E_{z} & =c^{2}\left(\sqrt{\frac{B_{z}}{B_{0}}} \partial_{x} B_{y}-\mu_{0} j_{z}\right) \\
\partial_{t} B_{y} & =\sqrt{\frac{B_{z}}{B_{0}}} \partial_{x} E_{z}-\partial_{z} E_{x}+E_{x} \frac{\partial_{z} B_{z}}{2 B_{z}}
\end{aligned}
$$

We use two fluids to describe the electrons, and let the subscript $s=M$ denote magnetospheric electrons while $s=I$ denotes the ionospheric population. The perpendicular motion of the electrons is given by the $\mathbf{E} \times \mathbf{B}$ drift and can be neglected, since it gives no contribution to the current. The motion along the field lines of an electron component with density $n_{s}$ and velocity $v_{s}$ is described by the continuity equation

$$
\partial_{t} n_{s}=-\partial_{z} n_{s} v_{s}+n_{s} v_{s} \frac{\partial_{z} B_{z}}{B_{z}}
$$

and the equation of motion. We assume that in a static equilibrium with density $n_{0 s}$ and temperature $T_{s}$, the pressure gradients $\partial_{z} n_{0 s} T_{s}$ are balanced by external forces $F_{0 s}$, that is, $F_{0 s}=\partial_{z} n_{0 s} T_{s}$. The electron velocity $v_{s}$ is then determined by the equation

$$
\partial_{t} v_{s}=-v_{s} \partial_{z} v_{s}-\frac{e}{m_{e}} E_{z}-\frac{\partial_{z} n_{s} T_{s}}{n_{s} m_{e}}+\frac{F_{0 s}}{n_{0 s} m_{e}} .
$$

The ion motion along the field lines is neglected, and we assume that the ion velocity $u_{y}$ in the $y$ direction is given by the $\mathbf{E} \times \mathbf{B}$ drift. We will consider the ions to be cold, which implies that the kinetic effects on Alfvén wave propagation are neglected, although they may be significant at altitudes above a few $R_{E}$. Including a prescribed mechanical force $F_{y}$ that drives the generator, the $y$ component of the equation of motion for the ions is

$$
d_{t} u_{y}=-\frac{e}{m_{i}} u_{x} B_{z}+\frac{F_{y}}{n_{i} m_{i}} .
$$

Inserting $u_{y}=-E_{x} / B_{z}$ allows us to calculate the ion velocity $u_{x}$ in the $x$ direction as

$$
u_{x}=\frac{m_{i}}{e B_{z}^{2}} \frac{\partial_{t} E_{x}+\frac{B_{z}}{n_{i} m_{i}} F_{y}}{1-\frac{m_{i}}{e B_{z}^{2}} \sqrt{\frac{B_{z}}{B_{0}}} \partial_{x} E_{x}} .
$$

The perpendicular current, which essentially is the ion polarization current, can then be written as

$$
\mu_{0} j_{x}=V_{A}^{-2} \frac{\partial_{t} E_{x}+\frac{B_{z}}{n_{i} m_{i}} F_{y}}{1-\frac{m_{i}}{e B_{z}^{2}} \sqrt{\frac{B_{z}}{B_{0}}} \partial_{x} E_{x}}
$$

where $V_{A}=B_{z} / \sqrt{\mu_{0} n_{i} m_{i}}$ is the Alfvén velocity. Inserting this in Ampere's law, we find

$$
\partial_{t} E_{x}=-\mathrm{A}^{2} c^{2}\left(\partial_{z} B_{y}-B_{y} \frac{\partial_{z} B_{z}}{2 B_{z}}\right)-\left(1-\mathrm{A}^{2}\right) \frac{B_{z}}{n_{i} m_{i}} F_{y}
$$

where

$$
\mathrm{A}^{2}=V_{A}^{2} \frac{1-\frac{m_{i}}{e B_{z}^{2}} \sqrt{\frac{B_{z}}{B_{0}}} \partial_{x} E_{x}}{c^{2}+V_{A}^{2}\left(1-\frac{m_{i}}{e B_{z}^{2}} \sqrt{\frac{B_{z}}{B_{0}}} \partial_{x} E_{x}\right)}
$$

We now introduce new dimensionless independent variables through

$$
\mathrm{x}=\frac{\Omega_{i}}{c} x, \quad \mathrm{z}=\frac{\Omega_{i}}{c} z, \quad \mathrm{t}=\Omega_{i} t
$$

where $\Omega_{i}=e B_{0} / m_{i}$, and the dimensionless fields

$$
\begin{aligned}
\mathrm{E}_{\mathrm{x}} & =E_{x} / \sqrt{c^{2} B_{0} B_{z}} \\
\mathrm{E}_{\mathrm{z}} & =E_{z} /\left(c B_{0}\right) \\
\mathrm{B} & =B_{y} / \sqrt{B_{0} B_{z}} \\
\mathrm{n}_{\mathrm{s}} & =n_{s} m_{i} /\left(\varepsilon_{0} B_{0} B_{z}\right), \\
\mathrm{T}_{\mathrm{s}} & =T_{s} /\left(m_{e} c^{2}\right) .
\end{aligned}
$$


We also define

$$
\begin{aligned}
\mathrm{j}_{\mathrm{s}} & =\mathrm{n}_{\mathrm{s}} \frac{v_{s}}{c}=-\frac{\mu_{0} c}{\Omega_{i} B_{z}} j_{s}, \\
\mathrm{~N}_{\mathrm{s}} & =\mathrm{n}_{\mathrm{s}}-\mathrm{n}_{0 \mathrm{~s}}, \\
\mathrm{~F} & =\frac{F_{y}}{e n_{i} c \sqrt{B_{0} B_{z}}} .
\end{aligned}
$$

We can then collect equations (2), (3), (4), and (8) in the form

$$
\begin{aligned}
\partial_{\mathrm{t}} \mathrm{E}_{\mathrm{x}} & =-\mathrm{A}^{2} \partial_{\mathrm{z}} \mathrm{B}-\left(1-\mathrm{A}^{2}\right) \mathrm{F} \\
\partial_{\mathrm{t}} \mathrm{E}_{\mathrm{z}} & =\frac{B_{z}}{B_{0}}\left(\partial_{\mathrm{x}} \mathrm{B}+\mathrm{j}_{\mathrm{M}}+\mathrm{j}_{\mathrm{I}}\right) \\
\partial_{\mathrm{t}} \mathrm{B} & =\partial_{\mathrm{x}} \mathrm{E}_{\mathrm{z}}-\partial_{\mathrm{z}} \mathrm{E}_{\mathrm{x}} \\
\partial_{\mathrm{t}} \mathrm{N}_{\mathrm{s}} & =-\partial_{\mathrm{z}} \mathrm{j}_{\mathrm{s}} \\
\partial_{\mathrm{t}} \mathrm{j}_{\mathrm{s}} & =-\frac{m_{i}}{m_{e}} \mathrm{n}_{\mathrm{s}} \mathrm{E}_{\mathrm{z}}-\partial_{\mathrm{z}}\left(\mathrm{N}_{\mathrm{s}} \mathrm{T}_{\mathrm{s}}+\frac{\mathrm{j}_{\mathrm{s}}^{2}}{\mathrm{n}_{\mathrm{s}}}\right)+\mathrm{N}_{\mathrm{s}} \frac{\partial_{\mathrm{z}} \mathrm{n}_{0 \mathrm{~s}} \mathrm{~T}_{\mathrm{s}}}{\mathrm{n}_{0 \mathrm{~s}}} .
\end{aligned}
$$

Here it may be useful to point out some physical processes and how they are described by equations (13). Eliminating $B$ between the first and third equations of (13), we obtain

$$
\left(\partial_{t}^{2}-A^{2} \partial_{z}^{2}\right) E_{x}+A^{2} \partial_{z} \partial_{x} E_{z}=-\left(1-A^{2}\right) \partial_{t} F,
$$

which describes driven shear Alfvén waves. While $F$ is growing during the early stages of our simulations, the dynamics will mainly be determined by (14), and when $\mathrm{F}$ becomes stationary, the Alfvén waves are decoupled from the force. If we assume a stationary state and eliminate $B$ from the first two equations of (13) we find, with $\mathrm{j}=\mathrm{j}_{\mathrm{M}}+\mathrm{j}_{\mathrm{l}}$,

$$
\partial_{z} j=-\left(1-A^{-2}\right) \partial_{x} F .
$$

Equation (15) describes an MHD generator. The shear in the mechanical force generates a field-aligned current. During the later stages of our simulations the current from (15) determines $E_{z}$ via the momentum equations, and in the electrostatic limit, $\partial_{z} E_{x}=\partial_{x} E_{z}$ gives the perpendicular electric field.

The equations of (13) describe the propagation of shear Alfvén waves and the formation of electrostatic structures but also high-frequency waves such as electrostatic oscillations at the plasma frequency $\omega_{p}$ and electromagnetic radiation. These high-frequency modes will cause severe problems if we try to integrate the equations with a time step $\Delta t \gg \omega_{p}^{-1}$ and a perpendicular resolution $\Delta x \ll c \Delta t$, suitable for the slow, shortscale processes we are interested in. In related numerical models [e.g., Goertz and Boswell, 1979; Lysak and Dum, 1983; Streltsov and Lotko, 1999] this problem is handled by neglecting the parallel displacement current, $\varepsilon_{0} \partial_{t} E_{z}$. If we in Ampere's law replace $\varepsilon_{0}$ by a dielectric tensor according to

$$
\varepsilon_{0}\left(\begin{array}{ccc}
1 & 0 & 0 \\
0 & 1 & 0 \\
0 & 0 & \varepsilon_{z}
\end{array}\right),
$$

where $\varepsilon_{z}=1$ in a normal vacuum, the second equation in (13) becomes

$$
\partial_{t} \mathrm{E}_{\mathbf{z}}=\frac{B_{z}}{\varepsilon_{z} B_{0}}\left(\partial_{\times} \mathrm{B}+\mathrm{j}_{\mathrm{M}}+\mathrm{j}_{\mathrm{I}}\right) .
$$

We see that neglecting the parallel displacement current corresponds to $\varepsilon_{z} \rightarrow 0$, or $\omega_{p z}^{2}=\omega_{p}^{2} / \varepsilon_{z} \rightarrow \infty$, which means that the current is assumed to adjust instantaneously to any changes in the magnetic field. When $B_{y}$ varies on a perpendicular scale comparable to the electron inertial length $\lambda_{i}=c / \omega_{p}$, it becomes difficult to justify this approximation, since the time it takes to build up a strong current increases as the plasma density decreases.

Rather than neglecting the displacement current by letting $\omega_{p z} \rightarrow \infty$, we will here use a less drastic method. We will instead increase the vacuum polarizability $\varepsilon_{z}$ and slow down the plasma oscillations to a frequency that permits the numerical integration to converge. We choose

$$
\varepsilon_{z}=\max \left(\omega_{p} \Delta t, 1\right),
$$

which allows us to resolve the normal plasma oscillations whenever $\omega_{p} \Delta t \lesssim 1$. When $\omega_{p} \Delta t>1$, the frequency of the plasma oscillations is brought down to $\omega_{p z} \approx \Delta t^{-1}$, which can be handled by the numerical integration. As long as the timescales we are interested in are well resolved by our $\Delta t$, it should not matter whether the plasma frequency is $\omega_{p}$ or $\omega_{p z}$ since they are both very high compared to the frequencies of interest. Notice that the phase velocity of electromagnetic radiation in the ordinary mode is also reduced to $c_{z}=c / \sqrt{\varepsilon_{z}}$. This means that the perpendicular resolution is improved to $\Delta x \sim c_{z} \Delta t$, which is comparable to $\lambda_{i}=c / \omega_{p}=c_{z} / \omega_{p z}$. When an anisotropic vacuum is introduced with a dielectric constant defined as in (18), the set of equations (13) becomes very convenient for numerical integration. These equations are integrated by a fully implicit method. Further details about the numerical method are given in Appendix A.

\subsection{Model Parameters and Boundary Conditions}

Although the model contains several parameters that can be varied, we will use a single set of values for most of them in this first study. We simulate a region $-L_{x}<x<L_{x}$, where $L_{x}$ is $500 \mathrm{~km}$ at the generator and $21.1 \mathrm{~km}$ in the ionosphere. The length of the simulation box is $L_{z}=3 \times 10^{4} \mathrm{~km}$, which is a few $R_{E}$ less than the distance from the ionosphere to the equatorial plane along a typical auroral field line. Since we neglect kinetic effects, the dispersion of short perpendicular wavelength Alfvén waves at higher altitudes is not described in detail by our model [Lysak and Lotko, 1996]. This shortening will lead to slightly shorter particle and Alfvén wave transit times but has little effect on the overall physics. Shortening the system saves 
computer time and makes the interesting parts of the resulting plots easier to read by minimizing the featureless section between the generator and the acceleration region.

The magnetic field strength along an auroral field line is rather well approximated by

$$
B_{z}(z)=B_{0} \exp \left\{z^{2} /\left[L_{z}^{2} \ln \left(B_{I} / B_{0}\right)\right]\right\},
$$

where we will use the ionospheric field strength $B_{I}=$ $56 \mu \mathrm{T}$ and $B_{0}=0.1 \mu \mathrm{T}$. This magnetic field model, which is justified by noticing that a logarithmic plot of the dipolar field strength on auroral latitudes versus $z$ resembles a parabola, implies that the distance between field lines increases by $\sqrt{B_{I} / B_{0}} \approx 23.6$ from the ionosphere to the generator.

The force $F_{y}$ applied in the generator region has the form

$$
F_{y}(x, z, t)=F_{0} \frac{\exp \left(-z^{2} / L_{z G}^{2}\right)}{\cosh ^{2}\left(x / L_{x G}\right)} \frac{t^{2}}{t^{2}+t_{G}^{2}}
$$

where $L_{z G}=6 \times 10^{3} \mathrm{~km}$ and $L_{x G}=50 \mathrm{~km}$. The maximum force $F_{0}$ will be $1.5 \times 10^{-17} \mathrm{~N} \mathrm{~m}^{-3}$, and in this study we will use the generator timescale $t_{G}=10 \mathrm{~s}$.

The density of the magnetospheric electrons is initially set to

$$
n_{0 M}(z)=\left[1+\frac{B_{z}}{B_{I}}\left(\frac{B_{z}}{B_{0}}\right)^{\frac{1}{4}}\right] 3 \times 10^{5} \mathrm{~m}^{-3},
$$

which is roughly constant down to below the acceleration region and then increases to $1.76 \times 10^{6} \mathrm{~m}^{-3}$ near the ionosphere. The temperature of the magnetospheric electron component is kept constant at $T_{M}=1 \mathrm{keV}$.

In terms of the height $h=L_{z}-z$ above the lower boundary, the density of ionospheric electrons is given by

$$
n_{0 I}(h)=\left\{\exp \left[\frac{-h}{H\left(1+h / 5 R_{E}\right)}\right]+10^{-8}\right\} 10^{9} \mathrm{~m}^{-3} .
$$

Here, $H=400 \mathrm{~km}$ is the scale height in the ionosphere, but the effective scale height increases slightly with altitude. The temperature of the ionospheric electrons is set to $T_{I}(h)=0.1 \mathrm{eV} \sqrt{n_{0 I}(0) / n_{0 I}(h)}$, with an upper limit of $100 \mathrm{eV}$.

The lower ionosphere is represented by a heightintegrated ionospheric Pedersen conductivity, which we take to be $\Sigma_{P}=10 \Omega^{-1}$. Integrating Ohm's law and Ampere's law across the ionospheric height leads to the condition

$$
B_{y}\left(L_{z}\right)=\mu_{0} \Sigma_{P} E_{x}\left(L_{z}\right)
$$

at the ionospheric boundary. For precipitating electrons $\left(\mathrm{j}_{\mathrm{s}}>0\right.$ ) we demand $\partial_{z} \mathrm{j}_{\mathrm{s}}=0$ at $z=L_{z}$, which also implies $\partial_{t} N_{s}\left(L_{z}\right)=0$. However, we do not allow elec- trons to flow up from the boundary into the simulation box, since that would add momentum to the system in an uncontrolled way. Hence we set $\mathrm{j}_{\mathrm{s}}\left(L_{z}\right)=0$ and $\partial_{z} \mathrm{~N}_{\mathrm{s}}\left(L_{z}\right)=0$ when $\mathrm{j}_{\mathrm{s}}<0$ inside the boundary. No ionospheric boundary condition is needed for $E_{z}$.

Since the force $F_{y}$ is symmetric around the equator, we assume that $E_{x}$ and the densities $n_{s}$ also are symmetric. Their boundary conditions at $z=0$ are thus $\partial_{z} E_{x}=\partial_{z} N_{s}=0$. The remaining fields are then antisymmetric, so we set $E_{z}=B=j_{s}=0$ at the equatorial plane. The fields are all set to zero on the boundaries at $x= \pm L_{x}$.

\section{Results}

To illustrate the response of the auroral plasma to a force applied in the equatorial region, we will show plots of selected fields at various times. The force $F_{y}$ is given by (20) with $F_{0}=1.5 \times 10^{-17} \mathrm{~N} \mathrm{~m}^{-3}$ and $t_{G}=10 \mathrm{~s}$. When this force starts to pull the equatorial plasma in the $y$ direction, the divergence of the perpendicular current causes a charge separation that sets up an electric field $\left(E_{x}, E_{z}\right)$. The growth of $E_{x}$ and the current $j_{z}$ driven by $E_{z}$ are coupled via the magnetic perturbation $B_{y}$.

Figures $2 \mathrm{a}-2 \mathrm{~d}$ show perspective plots of these fields $1 \mathrm{~s}$ after the force has been turned on. The fields in physical units are plotted as functions of the fieldaligned coordinates $(x, h)$, and the distances given on the $x$ axis refer to the ionospheric boundary $(h=0)$. We show only $|x|<10 \mathrm{~km}$, although the simulation covers $\pm 21 \mathrm{~km}$. The perpendicular electric field is still confined to the equatorial region and is roughly proportional to $F_{y}$. There are peaks in $B_{y}$ and $E_{z}$ at the altitude where $\partial_{z} E_{x}$ is large. A small $E_{z}$ is seen at altitudes around $7200 \mathrm{~km}$, where the Alfvén velocity reaches its maximum value of $\sim 1.1 \times 10^{8} \mathrm{~m} \mathrm{~s}^{-1}$. A significant current density, which causes a noticeable magnetic perturbation, is already starting to build up near the ionospheric boundary. Considering that the time it takes an Alfvén wave to propagate through the system,

$$
t_{L_{z}}=\int_{0}^{L_{z}} \frac{d z}{c \mathrm{~A}}
$$

is about $3.6 \mathrm{~s}$, it may be surprising that information about the generator has already reached the ionosphere. However, signals can also propagate with the sound speed $1.87 \times 10^{7} \mathrm{~m} \mathrm{~s}^{-1}$ of the magnetospheric electrons. By combining the two modes of propagation, a precursor can reach the ionosphere in $\sim 1 \mathrm{~s}$. Other simulations ran with $t_{G}=0.1 \mathrm{~s}$ show that the main pulse reaches the ionosphere at $t \sim t_{L_{z}}$ as expected.

In Figures 3a-3d we show the fields after $10 \mathrm{~s}$, when the force has grown to half its final value. The fields are now much more concentrated to the lower part of the flux tube. In particular, the magnetic perturbation $B_{y}$ and the field-aligned current density $j_{z}=j_{M}+j_{I}$ 
reach their maxima at the ionospheric boundary where the background magnetic field is strongest. The perpendicular electric field has a more complicated structure, which results from the combination of the growing pulse coming down from the generator and the reflected pulse from the ionosphere. The field-aligned electric field is concentrated to altitudes around $7000 \mathrm{~km}$, which is roughly where the normalized density $n=n_{M}+n_{1}$ has its minimum.

After $60 \mathrm{~s}$ the generator force has reached $97.3 \%$ of its final strength, and the fields shown in Figures $4 \mathrm{a}-4 \mathrm{~d}$ have become rather stationary. The magnetic disturbance $B_{y}$ is roughly proportional to $\sqrt{B_{z}}$, and $j_{z}$ is proportional to $B_{z}$. This is what we expect, since (13) indicates that $\partial_{z} B=\partial_{z} j_{s}=0$ below the generator in a stationary state. The strongest $E_{x}$ are found near the acceleration region, between the peaks in $E_{z}$. Notice the ridges with $E_{x}>0$ at high altitudes, which indicate regions where the plasma flow is in a direction opposite to the applied force. In the upward current region there is a strong negative $E_{z}$ accelerating the precipitating magnetospheric electrons, but in the downward current region the sign of $E_{z}$ changes with altitude. At lower altitudes a positive $E_{z}$ accelerates electrons upward from the ionosphere. Above the acceleration region a nega- tive $E_{z}$ is required to slow the electrons down as the flux tube widens and $\mathrm{n}$ increases.

A persistent feature in our simulations is that the downward currents are narrower and more intense than the upward currents, as seen in Figure 4d. Even if we at present cannot explain the reasons for this difference, it is interesting to notice that a similar effect was observed by the Fast Auroral SnapshoT (FAST) satellite [Elpic et al., 1998].

Although our simulations are based on the electromagnetic fields, it is instructive to consider also the potentials. In our case there are two straightforward ways to calculate the scalar potential. Setting the potential to zero in the ionosphere at $x=-L_{x}$, we can integrate the electric field to obtairı

$$
\phi_{z}(x, z, t)=-\int_{-L_{x}}^{x} E_{x}\left(x^{\prime}, L_{z}\right) d x^{\prime}-\int_{L_{z}}^{z} E_{z}\left(x, z^{\prime}\right) d z^{\prime} .
$$

The scalar potential $\phi_{z}$ corresponds to a particular choice of gauge, in which the vector potential $\mathbf{A}=A_{x} \hat{\mathbf{x}}$ and the fields are given by $E_{x}=-\partial_{x} \phi_{z}-\partial_{t} A_{x}, E_{z}=$ $-\partial_{z} \phi_{z}$, and $B_{y}=\partial_{z} A_{x}$. Alternatively, we can also compute the potential by doing the integrals in the reverse order as
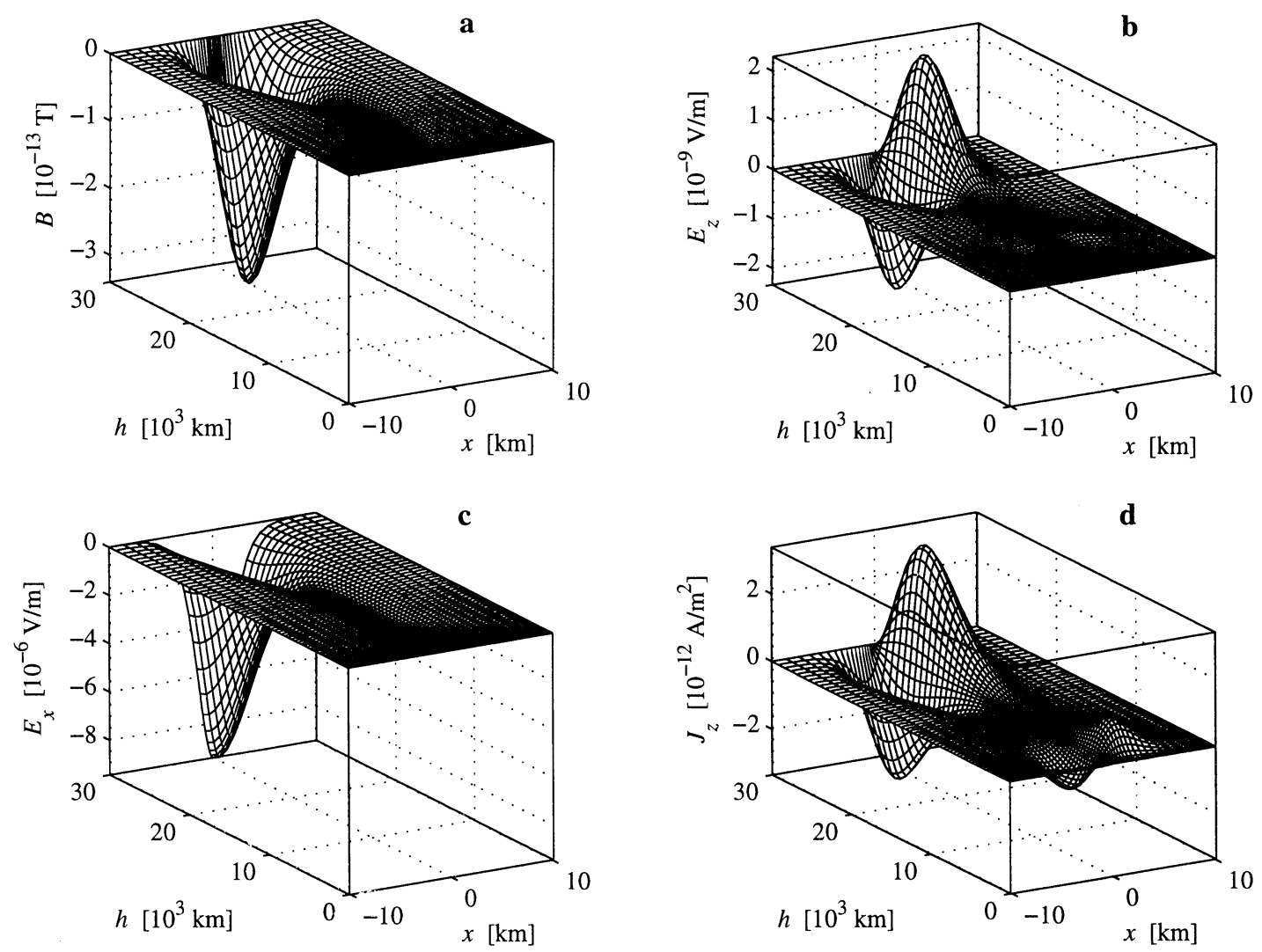

Figure 2. Perspective plots $1 \mathrm{~s}$ after the generator was started of (a) the magnetic field perturbation, (b) the parallel electric field, (c) the perpendicular electric field, and (d) the parallel current density. 

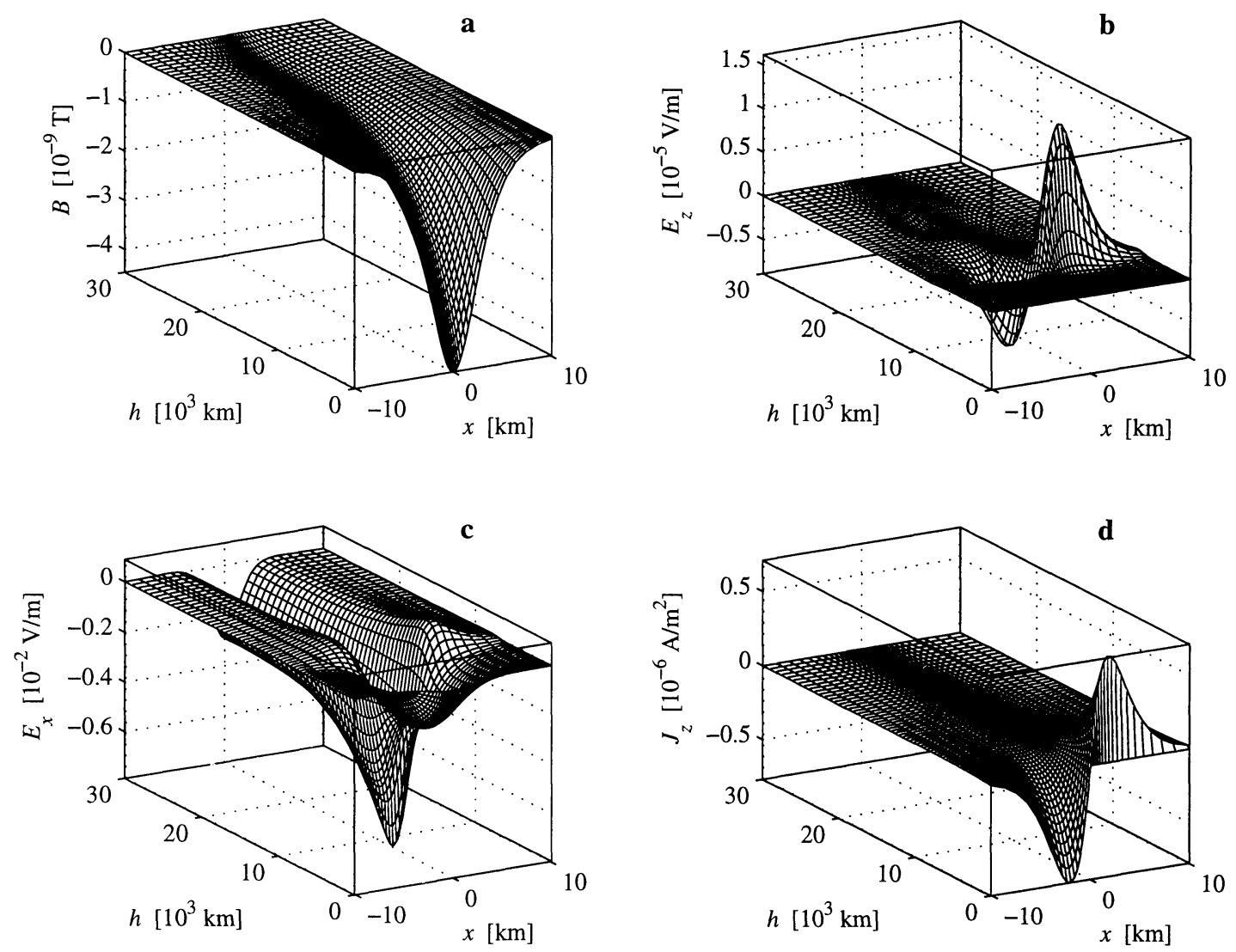

Figure 3. Perspective plots $10 \mathrm{~s}$ after the generator was started of (a) the magnetic field perturbation, (b) the parallel electric field, (c) the perpendicular electric field, and (d) the parallel current density.

$\phi_{x}(x, z, t)=-\int_{L_{z}}^{z} E_{z}\left(-L_{x}, z^{\prime}\right) d z^{\prime}-\int_{-L_{x}}^{x} E_{x}\left(x^{\prime}, z\right) d x^{\prime}$,

and in this gauge the fields are $E_{x}=-\partial_{x} \phi_{x}, E_{z}=$ $-\partial_{z} \phi_{x}-\partial_{t} A_{z}$, and $B_{y}=-\partial_{x} A_{z}$. In the stationary limit these two scalar potentials will become equal, but they include different parts of the time variations.

Figure 5a shows the shape of $\phi_{z}$ at $t=90 \mathrm{~s}$. The general shape of $\phi_{z}$ becomes similar to this a few seconds after the start, and it retains this shape while the magnitude grows. At $t=90 \mathrm{~s}$ the shape of $\phi_{x}$ shown in Figure $5 \mathrm{~b}$ is rather similar to that of $\phi_{z}$, indicating that the the field is mainly electrostatic. However, $\phi_{x}$ also contains a significant oscillating component. Figure 6 shows $\phi_{x}(x, 0, t)$ in the equatorial plane at times $t=71,76,81$, and $86 \mathrm{~s}$. We interpret this oscillation as a field line resonance, a standing shear Alfvén wave with period $t_{R} \approx 20 \mathrm{~s}$ that is excited by the growing force and then decouples from the generator when $F_{y}$ becomes stationary. The period $t_{R}$ remains constant when $t_{G}$ is varied, which confirms the resonant character of the oscillation. The time-dependent electric field associated with this Alfvén resonance will be mainly in the $x$ direction, and depending on the gauge chosen, it can be seen in either $A_{x}$ or $\phi_{x}$, but hardly at all in $\phi_{z}$.
The collisionless electrons in the auroral magnetosphere are in this study modeled by compressible fluids, and the acceleration of a fluid is in some respects different from the acceleration of noninteracting particles. Below the acceleration region, where the parallel electric field is short-circuited by the ionospheric plasma, the magnetospheric electron fluid must be accelerated by pressure gradients. Figure 7 shows the density perturbation profile at $t=90 \mathrm{~s}$ along a field line that reaches the ionosphere at $x=-1.3 \mathrm{~km}$, which is in the middle of the upward current region. As shown in Figure 7, the density of magnetospheric electrons piles up in the lower part of the acceleration region. The electrons are then accelerated to their final energy by the gradient below the density maximum. The final energy of the precipitating electrons depends on the density of the magnetospheric electrons at the ionospheric boundary. Figure 8 shows the kinetic energy of the magnetospheric electrons along the field line at $x=-1.3 \mathrm{~km}$ at $t=90 \mathrm{~s}$. With the model we use for $n_{0 M}$ the kinetic energy reached at the ionospheric boundary is very similar to the energy $e \Delta \phi$ gained by falling through the potential drop $\Delta \phi$ along the field line (compare Figure 5). We emphasize that although the compressibility of the magnetospheric electron fluid complicates the picture of the acceleration process, the potential drop 

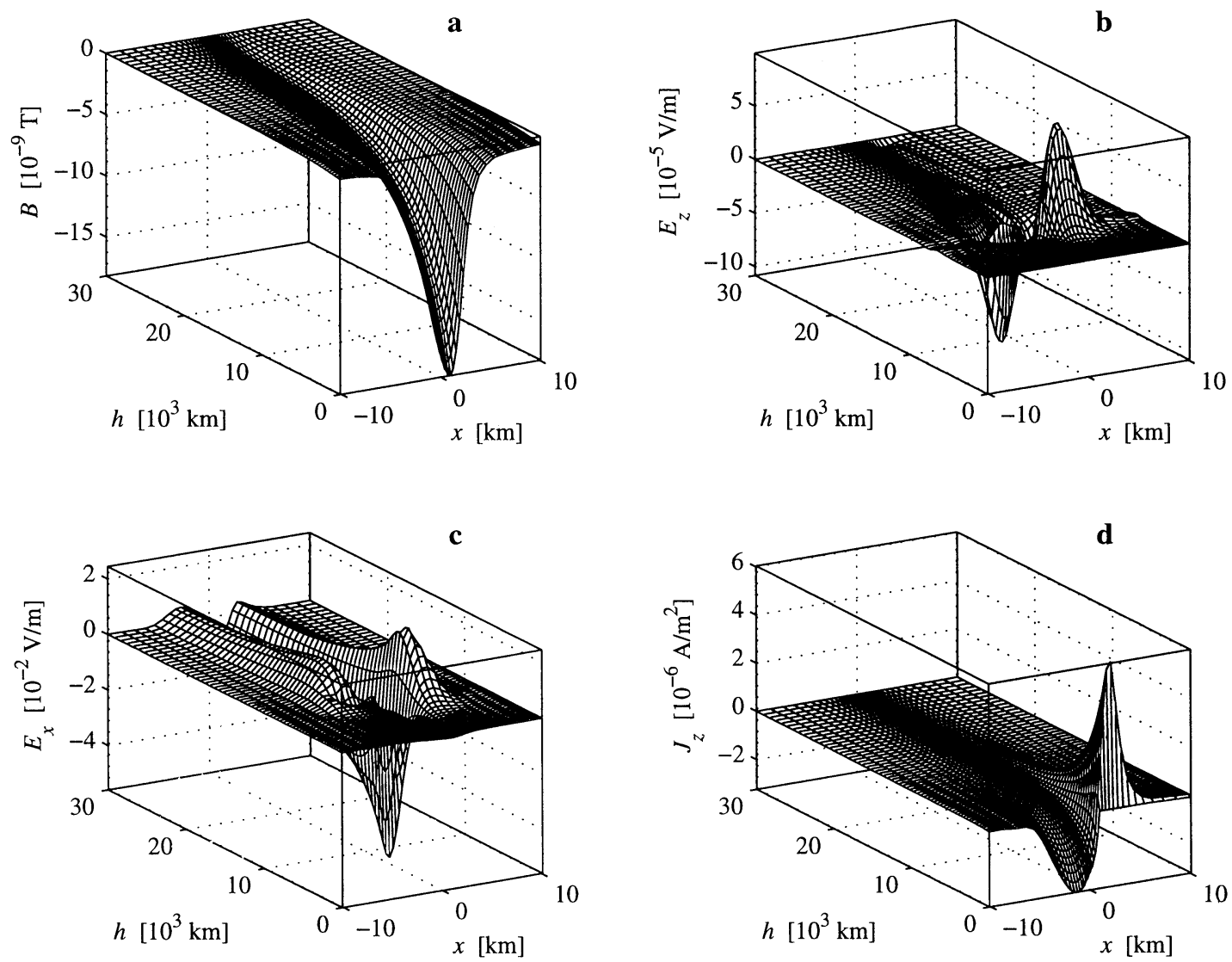

Figure 4. Perspective plots $60 \mathrm{~s}$ after the generator was started of (a) the magnetic field perturbation, (b) the parallel electric field, (c) the perpendicular electric field, and (d) the parallel current density.

in our simulations is supported by electron inertia and the low plasma density above the auroral ionosphere as discussed by Rönnmark [1999].

The density of ionospheric electrons is strongly depleted at altitudes around $9000 \mathrm{~km}$ in the upward current region. To prevent numerical problems with neg- ative densities (see Appendix A), we must limit this density reduction to $\sim 80 \%$. A density perturbation profile for the ionospheric electrons along the field line at $x=-1.3 \mathrm{~km}$ at $t=90 \mathrm{~s}$ is shown in Figure 9 .

In the downward current region the density of ionospheric electrons grows and builds up to a sharp peak
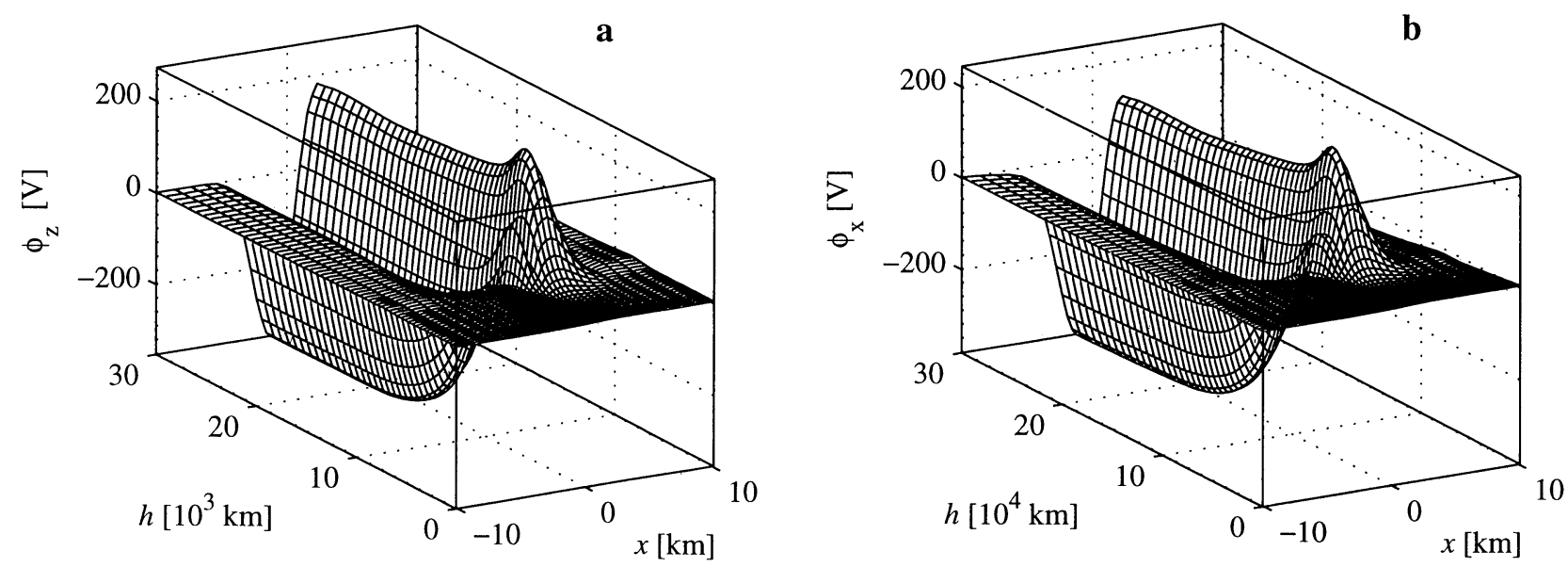

Figure 5. Perspective plots $90 \mathrm{~s}$ after the generator was started of (a) the scalar potential $\phi_{z}$ and (b) $\phi_{x}$. 


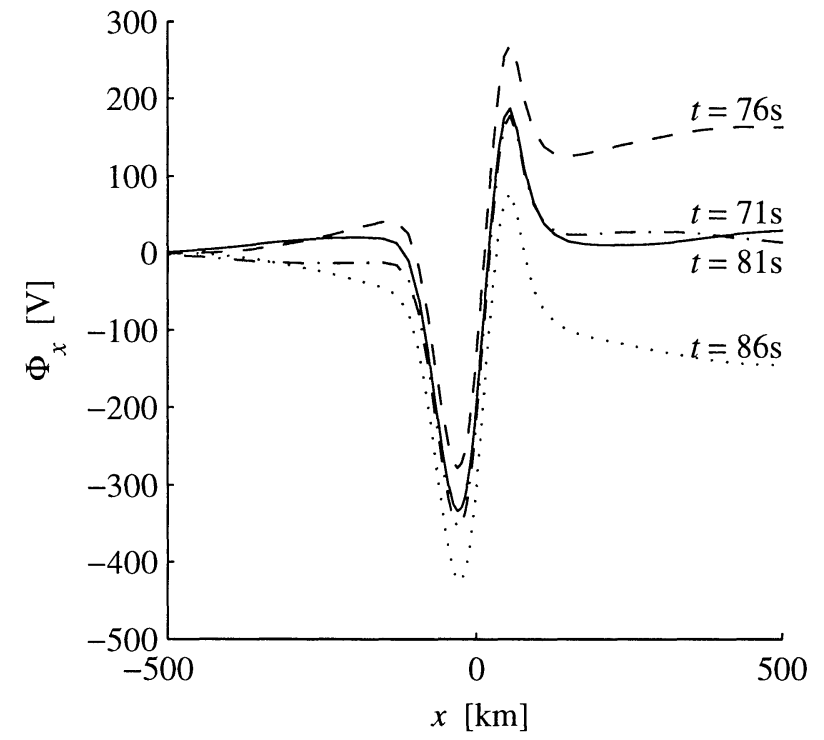

Figure 6. Cross section of the scalar potential $\phi_{x}$ in the equatorial plane at four times. The oscillation indicates that a field line resonance with a period $t_{R} \sim 20 \mathrm{~s}$ is excited.

near the reversal of $E_{z}$ at $h=8500 \mathrm{~km}$. This peak continues to move slowly upward throughout the simulation, while $N_{I}$ grows to almost $100 n_{0 I}$. The separation of the electrons into two fluids is not so easily justified in this region, and we are not convinced that the physics is described in detail by our model.

The total potential drop along a field line, given by $\Delta \phi(x, t)=\phi_{z}\left(x, L_{z}, t\right)-\phi_{z}(x, 0, t)$, is in Figure 10 plotted versus the current density $j_{M}\left(x, L_{z}, t\right)$ carried

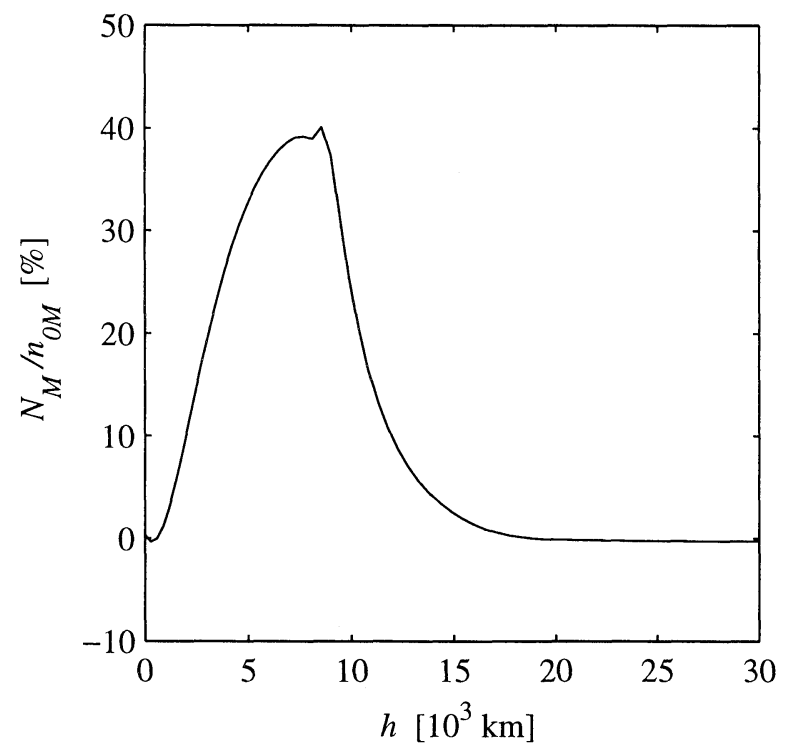

Figure 7. The relative density perturbation $N_{M} / n_{0 M}$ in the magnetospheric electrons along a field line at $x=$ $-1.3 \mathrm{~km}$ at $t=90 \mathrm{~s}$.

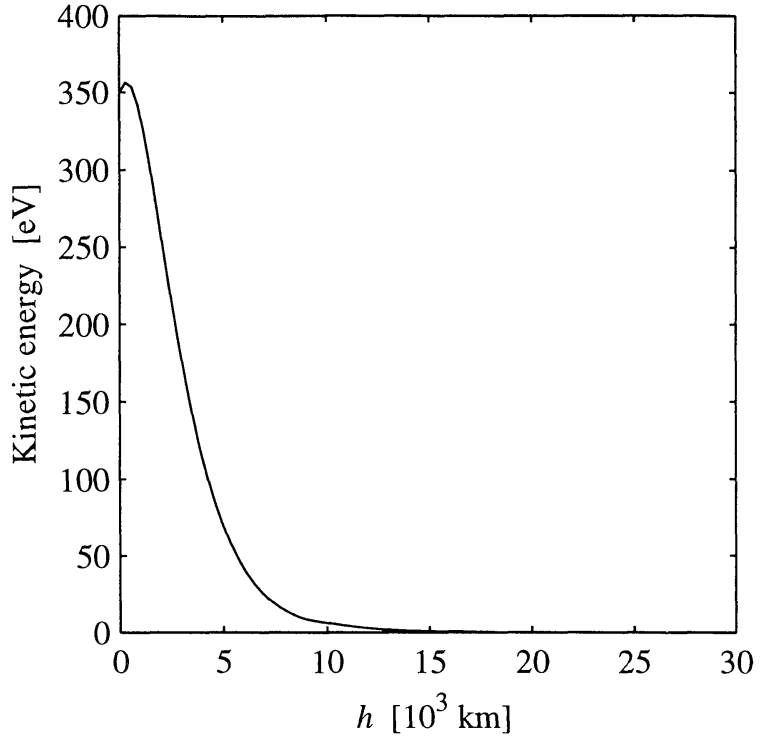

Figure 8. The kinetic energy of magnetospheric electrons along a field line at $x=-1.3 \mathrm{~km}$ at $t=90 \mathrm{~s}$.

into the ionosphere by the magnetospheric electrons. This is the current-voltage relation a spacecraft passing through the upward current region would observe at $t=90 \mathrm{~s}$. The crosses show values from the simulation, and a solid line shows the theoretical current-voltage relation derived by $R \ddot{n} n n$ mark [1999]

$$
\Delta \phi(x, t)=\frac{m_{e}}{2 e^{3} n^{2}} j_{M}^{2}
$$

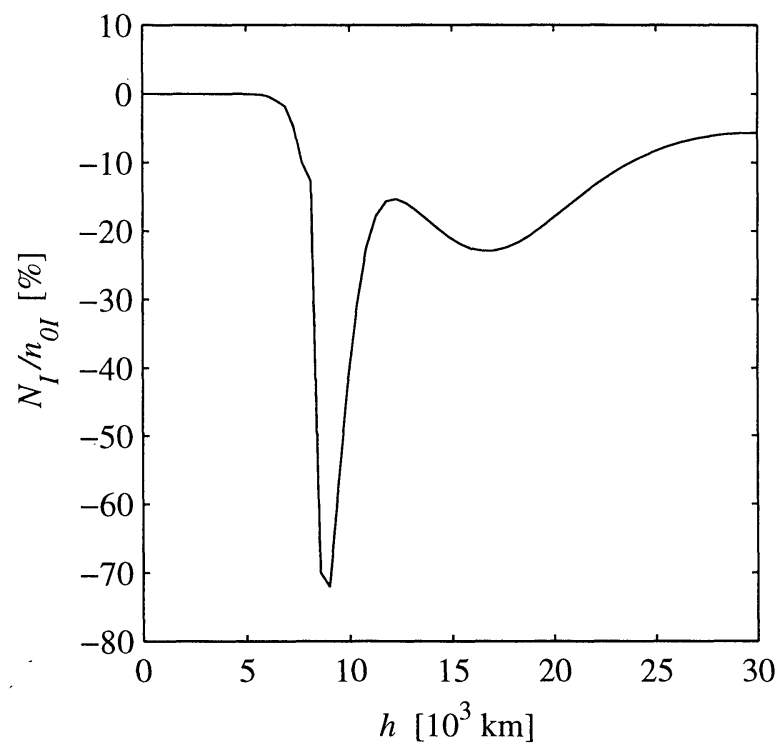

Figure 9. The relative density perturbation $N_{I} / n_{0 I}$ in the ionospheric electrons along a field line at $x=$ $-1.3 \mathrm{~km}$ at $t=90 \mathrm{~s}$. 


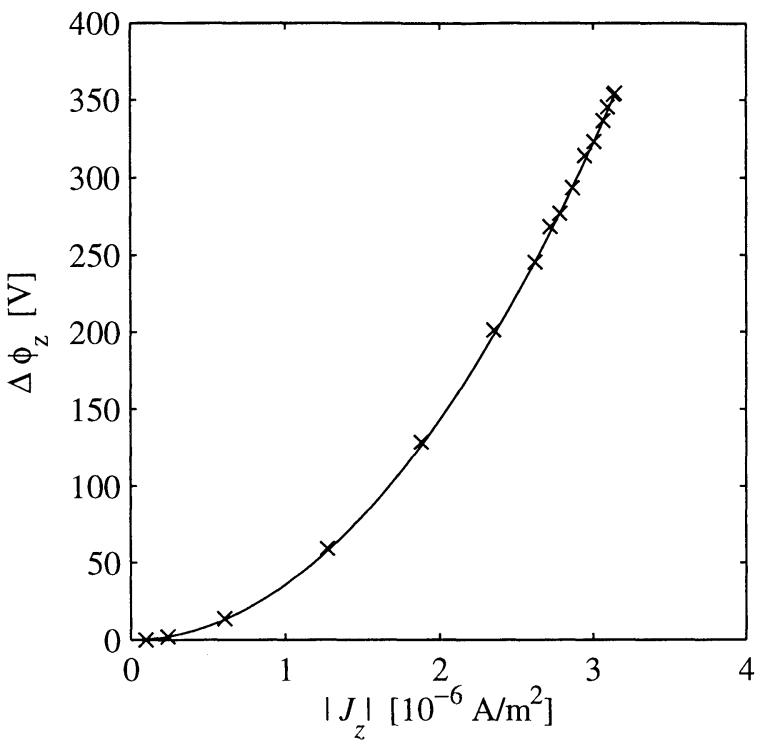

Figure 10. The current-voltage relation in the upward current region. The crosses show the simulation results, and the solid line is the theoretical result predicted by equation (27).

Using the density $n=1.76 \times 10^{6} \mathrm{~m}^{-3}$ for the magnetospheric electrons, we find $m_{e} /\left(2 e^{3} n^{2}\right)=3.576 \times$ $10^{13} \mathrm{~V} \mathrm{~A}^{-2}$, while a least squares fit to the crosses gives $3.593 \cdot 10^{13} \mathrm{~V} \mathrm{~A}^{-2}$. The agreement between the electrostatic fluid model and our numerical simulations is almost perfect, and our simulations strongly support that the self-consistent potential drop on auroral field lines is proportional to the square of the current density.

\section{Discussion}

The model developed in this study includes a generator that converts work done by equatorial, mechanical forces into electromagnetic power. The propagation of electromagnetic energy down to the acceleration region is described both during the dynamic stage dominated by Alfvén waves and during the electrostatic stage. In the acceleration region, electromagnetic power is converted back to mechanical energy in the form of an intense flux of precipitating energetic electrons.

There are several mechanisms that contribute to the appearance of parallel electric fields in shear Alfvén waves. At altitudes above $\sim 4 R_{E}$, parallel electric fields can be caused by finite Larmor radius effects [Hasegawa, 1976]. As discussed in section 2, these effects are not included in our model, but this omission is not likely to be important since most of the electron acceleration is expected to occur at lower altitudes. At these lower altitudes an $E_{z}$ is generated by electron inertia, that is, by terms proportional to the electron mass in the momentum equation

$$
d_{t} m_{e} v_{z}=\partial_{t} m_{e} v_{z}+m_{e} v_{z} \partial_{z} v_{z} \approx-e E_{z}
$$

In some studies [e.g., Goertz and Boswell, 1979; Streltsov and Lotko, 1999; Streltsov, 1999] only the partial time derivative $\partial_{t} m_{e} v_{z}$ is included, but the convective term is far more important in the auroral acceleration region for the slow, large-amplitude processes we consider. The convective term is included by Lysak and Dum [1983], but its effects are not seen since $n / B$ is constant in their model. As discussed by Rönnmark [1999], the term $m_{e} v_{z} \partial_{z} v_{z}$ alone can account for stationary, electrostatic potential drops of several kilovolts when the variation of $n / B$ along auroral field lines is taken into account. The importance of the convective term for electron acceleration by field line resonances has also recently been stressed by $\mathrm{A}$. N. Wright et al. (Parallel electric fields in the auroral acceleration region of field line resonances, submitted to Journal of Geophysical Research, 2000).

When describing the generator, we have assumed that the force $F_{y}$ is independent of the convection velocity $u_{y}=-E_{x} / B_{z}$ reached by the equatorial plasma. This corresponds to a pure current generator, and this idealized case was chosen for its simplicity. Mixtures between current and voltage generators can be modeled by allowing the force $F_{y}$ to depend on $u_{y}$, but it is not obvious how this dependence should be chosen. A selfconsistent treatment of the generator probably requires a three-dimensional simulation.

In the simulations presented here the auroral current sheets are $\sim 1 \mathrm{~km}$ thick and separated by $\sim$ $2 \mathrm{~km}$ at ionospheric altitude. These scales, which are determined by our choice of $F_{y}$, are typical for discrete arc systems, which may split up into several finer scale arcs when observed optically from the ground [Borovsky, 1993]. Our simulations show that the ionospheric resistance, which is essential for the large-scale magnetosphere-ionosphere coupling, is almost negligible for such small structures. This is also easily confirmed by an order of magnitude estimate, which shows that even if the ionospheric conductivity is as low as $1 \Omega^{-1}$ the potential drop in the ionosphere will be just a few volts for an auroral current density of $3 \mu \mathrm{A} \mathrm{m}^{-2}$ that yields a field-aligned potential drop $\sim 350 \mathrm{~V}$. This makes it very unlikely that enhancements of the ionospheric conductivity can have significant effects on the development of small-scale auroral structures.

The fluid model we use cannot fully describe the behavior of a collisionless plasma. As mentioned in section 3, the magnetospheric electrons must be accelerated by pressure gradients below the acceleration region. This limits the velocity they can reach to the sound velocity, which in our isothermal model equals their thermal velocity. The present version of the code behaves well for potential drops less than $\sim 400 \mathrm{~V}$, but for higher potentials it becomes difficult to control the density fluctuations.

The results presented in Figure 10 strongly support that the potential drop on auroral field lines is proportional to the square of the current. This forces 
us to reconsider the observations that have been interpreted as supporting a linear current-voltage relationship [Lyons et al., 1979; Weimer et al., 1987; Lu et al., 1991; Haerendel et al., 1994]:

$$
j_{z} \approx-K \Delta \phi
$$

These are event studies, and they contain no statistical evidence indicating whether the data are best fitted by a linear relation or some other function. In these studies the Lyons-Evans-Lundin constant $K$ [Fridman and Lemaire, 1980] is a free parameter, which varies by almost 2 orders of magnitude from event to event (and sometimes is adjusted during an event). Considering that the variation of the observed potential drop in each event is rather limited, it is probably possible to find a reasonable linear fit even if $\Delta \phi$ really is proportional to $j_{z}^{2}$

In a recent study of 22 events [Sakanoi et al., 1995] the latitudinal distribution of the upward current density was found to have an inverted-U shape, rather than the inverted- $\mathrm{V}$ shape of the potential. In many cases, $\Delta \phi / j_{z}$ was $\sim 5-10$ times larger at the center of the inverted-V potential than at the edges. Assuming that the plasma density is independent of latitude during each event, this result is expected if the potential drop is proportional to the square of the current. In our opinion, this study indicates that the observational support for a linear current-voltage relationship is rather weak.

There are also reasons to question the theoretical arguments in favor of a linear current-voltage relation. The kinetic models that predict a linear relation are not self-consistent but based on the adiabatic orbits of test particles. Initially, the particle distributions at the equatorial and ionospheric boundaries are specified in a state which we may assume to be charge neutral and without current. The adiabatic particle orbits will determine the distribution function, and hence the density, along the flux tube. An essentially arbitrary electrostatic potential is then prescribed, and new particle orbits are calculated by taking this potential into account. The particle distributions are then integrated to obtain the current, and the density can also be calculated. If the test particles in the model were real electrons, the densities calculated from the new orbits would imply large deviations from charge neutrality. Janhunen [1999] used this method to calculate the density in the presence of an upward electric field, and he found that for $e \Delta \phi \sim 2 T_{M}$ the density increase at low altitudes was comparable to the total equatorial density. A corresponding increase in the number of real electrons would cause a strong downward electric field, which is not included in the calculation of the particle orbits. As long as the particle distribution function calculated from the specified boundary conditions produces a charge density that is inconsistent with the potential, we have no reason to expect it to predict the correct current.
Even if kinetic test particle models cannot be used to obtain the current-voltage relation, they provide valuable information about the behavior of suprathermal particles in the auroral acceleration region. As long as the high-energy particles constitute a small fraction of the density, they will behave approximately as predicted by these models. In particular, the accelerated electrons below the parallel electric field will behave as test particles. Therefore the theoretical studies of velocity space boundaries between different particle populations, and the observations that confirm these theories, are not much affected by the lack of self-consistency. Some examples of such studies are discussed in the review by Lundin and Eliasson [1991].

In the literature, auroral electron acceleration by an electrostatic field has usually been treated as entirely separated from acceleration by shear Alfvén waves. Speaking about Alfvén wave models, Lysak and Dum [1983, p. 365] stated that "One problem with such models is that they do not possess a steady state parallel electric field to accelerate particles." and it seems that this opinion implicitly has been shared by most workers in this field. However, our simulations demonstrate that shear Alfvén waves build up an electric field that becomes electrostatic when the driving force is stationary. Even in the absence of collisions, there is a smooth transition from driven Alfvén waves to an electrostatic field maintained by a magnetospheric generator.

\section{Appendix A: Numerical Method}

The set of equations in (13) is solved by a fully implicit method, based on factorization of the twodimensional spatial differential operators. Algorithms of this type are discussed by, for example, Degrez [1992]. If we for notational convenience collect all the fields in a vector $\mathbf{U}=\left(\mathrm{E}_{\mathrm{x}}, \mathrm{E}_{\mathrm{z}}, \mathrm{B}_{\mathrm{y}}, \mathrm{N}_{\mathrm{s}}, \mathrm{j}_{\mathrm{s}}\right)^{T}$, we can after time discretization sum up (13) in the form

$$
\mathbf{U}(t+\Delta t)=\mathbf{U}(t)+\Delta t\{\mathbf{A}(\mathbf{U}(t+\Delta t / 2))+\mathbf{G}\},
$$

where $\mathbf{A}(\mathbf{U})$ represents the $\mathbf{U}$ dependence of the right hand side of (13) and $\mathbf{G}$ represents some inhomogeneous terms involving the external force $F$ and the densities $\mathrm{n}_{0 \mathrm{~s}}$. We now linearize $\mathbf{A}$ in $\mathbf{U}(t+\Delta t)$ by a Taylor expansion:

$$
\begin{aligned}
& \mathbf{A}(\mathbf{U}(t+\Delta t / 2)) \\
\approx & \mathbf{A}(\mathbf{U}(t))+\frac{1}{2}[\mathbf{U}(t+\Delta t)-\mathbf{U}(t)] \cdot \partial_{\mathbf{U}} \mathbf{A}(\mathbf{U}(t)) \\
= & \frac{1}{2}[\mathbf{U}(t+\Delta t)+\mathbf{U}(t)] \cdot \partial_{\mathbf{U}} \mathbf{A}(\mathbf{U}(t))
\end{aligned}
$$

where the second step follows from the homogeneous properties of $\mathbf{A}$. This results in a linear set of equations:

$$
\left[\mathbf{I}-\frac{\Delta t}{2} \partial_{\mathbf{U}} \mathbf{A}\right] \cdot \mathbf{U}(t+\Delta t)=\left[\mathbf{I}+\frac{\Delta t}{2} \partial_{\mathbf{U}} \mathbf{A}\right] \cdot \mathbf{U}(t)+\Delta t \mathbf{G} .
$$


The operator $\partial_{\mathbf{U}} \mathbf{A}$, which contains both $\partial_{x}$ and $\partial_{z}$, is now split into two parts as $\partial_{\mathbf{U}} \mathbf{A}=\mathbf{X}+\mathbf{Z}$, where $\mathbf{X}$ contains only $\partial_{x}$ and $\mathbf{Z}$ contains only $\partial_{z}$. This decomposition is not unique, and it should be chosen in a way that makes the product $\mathbf{X} \cdot \mathbf{Z}$ as small and simple as possible. Neglecting a small term $\Delta t^{2} / 4 \mathbf{X} \cdot \mathbf{Z} \cdot[\mathbf{U}(t+\Delta t)-\mathbf{U}(t)]$, we then find

$$
\begin{aligned}
& {\left[\mathbf{I}-\frac{\Delta t}{2} \mathbf{X}\right] \cdot\left[\mathbf{I}-\frac{\Delta t}{2} \mathbf{Z}\right] \cdot \mathbf{U}(t+\Delta t)=} \\
& {\left[\mathbf{I}+\frac{\Delta t}{2} \mathbf{X}\right] \cdot\left[\mathbf{I}+\frac{\Delta t}{2} \mathbf{Z}\right] \cdot \mathbf{U}(t)+\Delta t \mathbf{G} .}
\end{aligned}
$$

Introducing $\mathbf{U}^{*}=[\mathbf{I}-\Delta t / 2 \mathbf{Z}] \cdot \mathbf{U}(t+\Delta t)$ as a new variable, we can solve (33) in two steps. First we solve

$$
\begin{aligned}
& {\left[\mathbf{I}-\frac{\Delta t}{2} \mathbf{X}\right] \cdot \mathbf{U}^{*}=} \\
& {\left[\mathbf{I}+\frac{\Delta t}{2} \mathbf{X}\right] \cdot\left[\mathbf{I}+\frac{\Delta t}{2} \mathbf{Z}\right] \cdot \mathbf{U}(t)+\Delta t \mathbf{G} .}
\end{aligned}
$$

for $\mathbf{U}^{*}$. Then we use

$$
\left[\mathbf{I}-\frac{\Delta t}{2} \mathbf{Z}\right] \cdot \mathbf{U}(t+\Delta t)=\mathbf{U}^{*}
$$

to solve for the fields at $t+\Delta t$. When the operators $\mathbf{X}$ and $\mathbf{Z}$ are expressed as centered finite differences, each of these two steps consists of solving a block-tridiagonal set of equations. The number of operations needed for this scales linearly with the mesh size $\left(N_{x} \times N_{z}\right)$ and hardly requires any extra memory, which makes this algorithm very efficient. The number of operations needed for a direct integration of (32) would typically be proportional to $\left(N_{x} \times N_{z}\right)^{2}$.

The simulations presented here were all performed on a rectangular, slightly nonuniform grid of size $\left(N_{x} \times\right.$ $\left.N_{z}\right)=(51 \times 120)$, and we used a time step $\Delta t=0.02 \mathrm{~s}$.

The algorithm described above is numerically stable, but the grid tends to become decoupled in the ionosphere where $E_{z}$ is small. To cure this, even and odd grid points are coupled by averaging the fields $E_{x}, n_{s}$, and $j_{s}$ as

$$
\mathbf{U}(z)=0.9 \mathbf{U}(z)+0.05[\mathbf{U}(z+\Delta z)+\mathbf{U}(z-\Delta z)] .
$$

Some extra precautions are needed to ensure that the code behaves well in simulations that should converge to a quasi-stationary state with potential drops of hundreds of volts. To prevent the densities from going to zero near boundaries where the current is forced to vanish, we add a source term of the form $-S(z) \mathrm{N}_{\mathrm{s}}$ to the continuity equations. The function $S(z)$ is exponentially small away from the boundaries, except that it in the upward current region includes a term $10^{-3}\left(n_{01} / n_{1}\right)^{3}$ which becomes significant for very small $n_{1}$. If the ionospheric electrons are allowed to become supersonic, steep density gradients will form in the lower part of the acceleration region, where their density already is reduced by typically $80 \%$. To prevent this from making the density negative, we transfer some momentum from ionospheric to magnetospheric electrons by subtracting a force $\propto 0.05 m_{e} v_{I}^{2} / T_{I}$ in the momentum equation for the ionospheric electrons, and we conserve total current by adding a corresponding term to the magnetospheric component.

Acknowledgments. Michel Blanc thanks Joachim Vogt and another referee for their assistance in evaluating this paper.

\section{References}

Birn, J., M. Hesse, G. Haerendel, W. Baumjohann, and K. Shiokawa, Flow braking and the substorm current wedge, J. Geophys. Res., 104, 19,895-19,903, 1999.

Block, L. P., Potential double layers in the ionosphere, Cosmic Electrodyn., 3, 349-376, 1972.

Borovsky, J. E., Auroral arc thickness as predicted by various theories, J. Geophys. Res., 98, 6101-6138, 1993.

Degrez, G., Implicit time-dependent methods for inviscid and viscous compressible flows, in Computational Fluid Dynamics, edited by J. F. Wendt, pp. 180-222, SpringerVerlag, New York, 1992.

Elpic, R. C., et al., The auroral current circuit and fieldaligned currents observed by FAST, Geophys. Res. Lett., 25, 2033-2036, 1998.

Fridman, M., and J. Lemaire, Relationship between auroral electron fluxes and field-aligned electric potential difference, J. Geophys. Res., 85, 664-670, 1980.

Ganguli, S. B., H. G. Mitchell, and P. J. Palmadesso, Plasma dynamics driven by finite-width current filament and $\mathrm{keV}$ potential drops in ionosphere-magnetosphere coupling, Geophys. Res. Lett., 20, 975-978, 1993.

Génot, V., P. Louarn, and D. Le Quéau, A study of the propagation of Alfvén waves in the auroral density cavities, $J$. Geophys. Res., 104, 22,649-22,656, 1999.

Goertz, C. K., and R.W. Boswell, Magnetosphere-ionosphere coupling, J. Geophys. Res., 84, 7239-7246, 1979.

Haerendel, G., An Alfvén wave model of auroral arcs, in High-Latitude Space Plasma Physics, edited by B. Hultqvist and T. Hagfors, pp. 515-535,Plenum, New York, 1983.

Haerendel, G., H. U. Frey, O. H. Bauer, E. Rieger, J. Clemmons, M. H. Boehm, D. D. Wallis, and H. Lühr, Inverted$\mathrm{V}$ events simultaneously observed with the Freja satellite and from ground, Geophys. Res. Lett., 21, 1891-1894, 1994.

Hasegawa, A., Particle acceleration by MHD surface wave and formation of aurora, J. Geophys. Res., 81, 5083-5090, 1976.

Janhunen, P., On the current-voltage relationship in fluid theory, Ann. Geophys., 17, 11-26, 1999.

Janhunen, P., and A. Olsson, The current-voltage relationship revisited: Exact and approximate formulas with almost general validity for hot magnetospheric electrons for bi-Maxwellian and kappa distributions, Ann. Geophys., 16, 292-297, 1998.

Kletzing, C. A., Electron acceleration by kinetic Alfvén waves, J. Geophys. Res., 99, 11,095-11,103, 1994.

Knight, S., Parallel electric fields, Planet. Space Sci., 21, 741-750, 1973.

Lu, G., P. H. Reiff, J. L. Burch, and J. D. Winningham, On the auroral current-voltage relationship, J. Geophys. Res., 96, 3523-3531, 1991.

Lundin, R., and L. Eliasson, Auroral energization processes, Ann. Geophys., 9, 202-223, 1991.

Lyons, L. R., D. S. Evans, and R. Lundin, An observed relation between magnetic field-aligned electric fields and 
downward electron energy fluxes in the vicinity of auroral forms, J. Geophys. Res., 84, 457-461, 1979.

Lysak, R. L., Feedback instability of the ionospheric resonant cavity, J. Geophys. Res., 96, 1553-1568, 1991.

Lysak, R.L., and C. Dum, Dynamics of magnetosphereionosphere coupling including turbulent transport, J. Geophys. Res., 88, 365-380, 1983.

Lysak, R.L., and W. Lotko, On the kinetic dispersion relation for shear Alfvén waves, J. Geophys. Res., 101, 50855094, 1996.

Mitchell, H. G., S. B. Ganguli, and P. J. Palmadesso, Diodelike response of high-latitude plasma in magnetosphereionosphere coupling in the presence of field-aligned currents, J. Geophys. Res., 97, 12,045-12,056, 1992.

Papadopolous, K., A review of anomalous resistivity for the ionosphere, Rev. Geophys., 15, 113-127, 1977.

Rönnmark, K., Electron acceleration in the auroral current circuit, Geophys. Res. Lett., 26, 983-986, 1999.

Sakanoi, T., H. Fukunishi, and T. Mukai, Relationship between field-aligned currents and inverted-V parallel potential drops observed at midaltitudes, J. Geophys. Res., 100, 19,343-19,360, 1995.
Streltsov, A. V., Dispersive width of the Alfvénic field line resonance, J. Geophys. Res., 104, 22,657-22,666, 1999.

Streltsov, A. V., and W. Lotko, Dispersive field line resonances on auroral field lines, J. Geophys. Res., 100, 19,457-19,472, 1995.

Streltsov, A. V., and W. Lotko, Small-scale "electrostatic" auroral structures and Alfvén waves, J. Geophys. Res., 104, 4411-4426, 1999.

Weimer, D. R., D. A. Gurnett, C. K. Goertz, J. D. Menietti, J. L. Burch, and M. Sugiura, The current-voltage relationship in auroral current sheets, J. Geophys. Res., 92, 187-194, 1987.

M. Hamrin and K. Rönnmark, Theoretical Space Physics, Umeå University, S-901 87 Umeå, Sweden. (maria. hamrin@space.umu.se; kjell.ronnmark@space.umu.se)

(Received March 6, 2000; revised May 25, 2000; accepted June 15, 2000.) 